\title{
A dusty benchmark brown dwarf near the ice line of HD 72946*
}

\author{
A.-L. Maire ${ }^{1, \star \star}$, J.-L. Baudino ${ }^{2}$, S. Desidera ${ }^{3}$, S. Messina ${ }^{4}$, W. Brandner ${ }^{5}$, N. Godoy ${ }^{6,7}$, F. Cantalloube ${ }^{5}$, \\ R. Galicher ${ }^{8}$, M. Bonnefoy ${ }^{9}$, J. Hagelberg ${ }^{10}$, J. Olofsson ${ }^{6,7}$, O. Absil ${ }^{1, \star \star \star}$, G. Chauvin ${ }^{9,11}$, \\ T. Henning ${ }^{5}$, and M. Langlois ${ }^{12}$ \\ (Affiliations can be found after the references)
}

Received 18 November 2019 / Accepted 4 December 2019

\begin{abstract}
Context. HD 72946 is a bright and nearby solar-type star hosting a low-mass companion at long period $(P \sim 16 \mathrm{yr})$ detected with the radial velocity (RV) method. The companion has a minimum mass of $60.4 \pm 2.2 M_{J}$ and might be a brown dwarf. Its expected semi-major axis of $\sim 243$ mas makes it a suitable target for further characterization with high-contrast imaging, in particular to measure its inclination, mass, and spectrum and thus definitely establish its substellar nature.

Aims. We aim to further characterize the orbit, atmosphere, and physical nature of HD 72946B.

Methods. We present high-contrast imaging data in the near-infrared with the Spectro-Polarimetric High-contrast Exoplanet REsearch (SPHERE) instrument. We also use proper motion measurements of the star from HIPPARCOS and Gaia.

Results. The SPHERE data reveal a point source with a contrast of $\sim 9$ mag at a projected separation of $\sim 235$ mas. No other point sources are detected in the field of view. By jointly fitting the RV, imaging, and proper motion data, we constrain all the orbital parameters of HD 72946B and assess a dynamical mass of $72.4 \pm 1.6 M_{J}$ and a semi-major axis of $6.45_{-0.07}^{+0.08}$ au. Empirical comparison of its SPHERE spectrum to template dwarfs indicates a spectral type of L5.0 \pm 1.5 . The $J-H 3$ color is close to the expectations of the DUSTY models and suggests a cloudy atmosphere. Comparison with atmospheric models of the spectrophotometry suggests an effective temperature of $\sim 1700 \mathrm{~K}$. The bolometric luminosity $\left(\log \left(L / L_{\odot}\right)=-4.11 \pm 0.10 \mathrm{dex}\right)$ and dynamical mass of HD 72946B are more compatible with evolutionary models for an age range of $\sim 0.9-3 \mathrm{Gyr}$. The formation mechanism of the companion is currently unclear as the object appears slightly away from the bulk of model predictions. HD 72946B is currently the closest benchmark brown dwarf companion to a solar-type star with imaging, RV, and proper motion measurements.
\end{abstract}

Key words. brown dwarfs - methods: data analysis - stars: individual: HD 72946 - planets and satellites: dynamical evolution and stability techniques: high angular resolution - techniques: image processing

\section{Introduction}

Dynamical mass measurements of brown dwarfs are a powerful test of their formation and evolution models. Most studies exploit brown dwarf binaries (e.g., Konopacky et al. 2010; Dupuy \& Liu 2017; Dieterich et al. 2018), which have likely formed by fragmentation of a collapsing cloud (e.g., Bate 2009). However, it is still unclear whether brown dwarfs found at close-in separations to stars form like stellar binaries or by disk gravitational instabilities (Boss 1997). In the past years, a few radial velocity (RV) surveys started to target stars with slow drifts to constrain the orbit and minimum mass of the suspected long-period companions (e.g., Bouchy et al. 2016; Sahlmann et al. 2011; Feroz et al. 2011). These surveys have shown a paucity of brown dwarf companions within $5 \mathrm{au}$ from the host stars with respect to planetary and stellar companions (the so-called "brown dwarf desert", see e.g., Grether \& Lineweaver 2006; Sahlmann et al. 2011; Ma \& Ge 2014). Nevertheless, Ma \& Ge (2014) found that their occurrence increases at larger separations when brown dwarf detections from various techniques are combined.

Using the ELODIE and SOPHIE instruments, Bouchy et al. (2016) reported a potential brown dwarf companion to the G5V

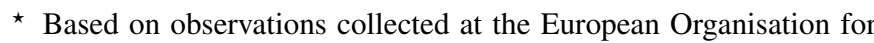
Astronomical Research in the Southern Hemisphere under ESO programme 0102.C-0781.

$\star \star$ F.R.S.-FNRS Postdoctoral Researcher.

$\star \star \star$ F.R.S.-FNRS Research Associate.
}

star HD 72946, located at $25.87 \pm 0.08 \mathrm{pc}$ (Gaia Collaboration 2016, 2018). The RV data cover a full orbit of HD 72946B, which allowed the authors to place good constraints on its orbit (period $P=15.93_{-0.13}^{+0.15} \mathrm{yr}$, eccentricity $e=0.495 \pm 0.006$, and periastron $\left.T_{0}[\mathrm{HJD}]=2455958 \pm 10\right)$. They derived a minimum dynamical mass of $60.4 \pm 2.2 M_{J}$ and an upper mass limit of $0.2 M_{\odot}$ from the analysis of the cross-correlation function of the star.

We present in this Letter the confirmation and characterization of the brown dwarf companion to HD 72946 with the Spectro-Polarimetric High-contrast Exoplanet REsearch (SPHERE) instrument and HIPPARCOS-Gaia proper motion measurements. We present an updated analysis of the properties of the host star in Sect. 2 and the SPHERE imaging observations in Sect. 3. We perform a joint orbital fit of the imaging, RV, and astrometric data and derive a dynamical mass for HD 72946B in Sect. 4. Section 5 discusses the spectral properties of the companion. Finally, we compare the physical and spectral properties of HD 72946B to model predictions in Sect. 6.

\section{Properties of the host star}

Bouchy et al. (2016) inferred from spectroscopic observations an effective temperature $T_{\text {eff }}=5686 \pm 40 \mathrm{~K}$, a surface gravity $\log g=4.50 \pm 0.06 \mathrm{dex}$, and a metallicity $[\mathrm{Fe} / \mathrm{H}]=0.11$ \pm 0.03 dex. The supersolar metallicity has been confirmed by 

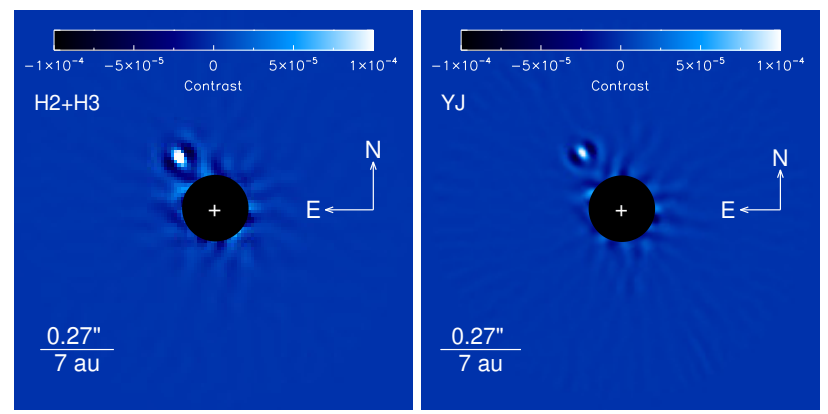

Fig. 1. SPHERE contrast images of HD 72946. The central regions of the images are numerically masked out to hide bright stellar residuals. The white crosses indicate the location of the primary star.

other studies (Aguilera-Gómez et al. 2018; Luck \& Heiter 2006; Casagrande et al. 2011, $0.15 \pm 0.06,0.16 \pm 0.04$, and $0.12 \mathrm{dex}$, respectively).

We derived the stellar age and mass from isochrones using the PARAM web interface ${ }^{1}$ (da Silva et al. 2006). We adopted the spectroscopic $T_{\text {eff }}$ and $[\mathrm{Fe} / \mathrm{H}]$ in Bouchy et al. (2016) with enlarged uncertainties to account for systematic errors. We also adopted the Gaia parallax and the $V$-band magnitude from HIPPARCos $(7.08 \pm 0.02 \mathrm{mag})$. This results in an age $1.712 \pm 1.684 \mathrm{Gyr}$, a mass $0.986 \pm 0.027 M_{\odot}$, and a radius $0.908 \pm 0.018 R_{\odot}$. Tighter constraints on the age from isochrones can be derived from the F8 comoving companion HD 72945 $(1.6 \pm 1.0 \mathrm{Gyr}$, Appendix A).

Lithium data $(\mathrm{A}(\mathrm{Li})=1.41,1.23,1.22 \pm 0.15$ dex, Luck 2017; Luck \& Heiter 2006; Ramírez et al. 2012) indicate an age older than that of the Hyades and similar to the open cluster NGC 752 (Sestito et al. 2004). The stellar kinematics suggest an age younger than the Sun, the UVW velocities being at the boundary of the kinematic space of young stars in Montes et al. (2001). Comparisons with stars with similar kinematics in Casagrande et al. (2011) indicated that it is unlikely that the star is older than $\sim 3$ Gyr and much younger than 0.5 Gyr.

We searched for archival photometric data to derive an age with gyrochronology, but we did not find suitable data (sampling, accuracy, blending with HD 72945, and/or calibration issues). Using the relations in Mamajek \& Hillenbrand (2008) and an averaged measured chromospheric activity of $-4.60 \mathrm{dex}$ (individual values $-4.54,-4.74 \pm 0.05,-4.66$, and $-4.47 \mathrm{dex}$, Rocha-Pinto et al. 2004; Bouchy et al. 2016; Gray et al. 2003; Boro Saikia et al. 2018), we derive a rotation period of $\sim 15 \mathrm{~d}$, which implies a gyrochronological age of $\sim 1$ Gyr. This is in between the loci of the Hyades (625-700 Myr) and NGC 752 (2000 Myr). The star has X-ray data from ROSAT, but is blended with HD 72945. However, X-ray activity is expected to correlate with chromospheric activity, so that it does not provide a fully independent age estimate. Assuming our derived stellar radius and an averaged measured projected rotational activity of $4.14 \mathrm{~km} \mathrm{~s}^{-1}$ (individual values $3.23,3.9 \pm 1$, and $5.3 \mathrm{~km} \mathrm{~s}^{-1}$, Martínez-Arnáiz et al. 2010; Bouchy et al. 2016; Luck 2017), we derive an upper limit for the rotation period of $12 \mathrm{~d}$, which implies a gyrochronological age younger than $1 \mathrm{Gyr}$ considering a $B-V$ color of 0.71 mag. Considering the large uncertainties in $v \sin i_{\star}$, the upper limit for the gyrochronological age could be as old as $1.5 \mathrm{Gyr}$. This means that our various age estimates agree overall. In the following, we choose to adopt an age range of $0.8-3 \mathrm{Gyr}$, with a most probable value of $1-2 \mathrm{Gyr}$.

http://stev.oapd.inaf.it/cgi-bin/param_1.3

\section{Observations and data analysis}

We observed HD 72946 on 2019 March 21 UT with the standard IRDIFS mode of SPHERE (Beuzit et al. 2019), which allows for simultaneous near-IR observations with IRDIS with the $H 23$ filter pair (Dohlen et al. 2008; Vigan et al. 2010) and the integral field spectrograph IFS in the $Y J$ bands (Claudi et al. 2008). The seeing and coherence time measured by the differential image motion monitor at $0.5 \mu \mathrm{m}$ were $0.5-0.7^{\prime \prime}$ and $6-8 \mathrm{~ms}$, respectively. The detector integration time was set to $16 \mathrm{~s}$, and 128 frames were recorded, amounting to a field rotation of $15.5^{\circ}$.

An apodized pupil Lyot coronagraph (Carbillet et al. 2011; Martinez et al. 2009) was used. We acquired data before and after the sequence to calibrate the flux of the images and the location of the star behind the coronagraph (Langlois et al. 2013). Night-time sky background frames were taken and additional daytime calibration performed following the standard procedure at ESO.

The data were reduced with the SPHERE Data Reduction and Handling software (v0.15.0, Pavlov et al. 2008) and custom routines for IFS data adapted from Mesa et al. (2015) and Vigan et al. (2015). This corrected for the cosmetics and instrument distortion, registered the frames, and normalized their flux. For IFS, it also performed the wavelength calibration and extracted the image cubes. Then, the data were analyzed with angular differential imaging (Marois et al. 2006) using three algorithms (Appendix B): ANDROMEDA, TLOCI, and PCA. Figure 1 shows the ANDROMEDA images.

The photometry and astrometry were extracted using three algorithms, but we chose to retain the TLOCI values (Table 1). The astrometry was calibrated following Maire et al. (2016), with pixel scales of $12.255 \pm 0.009$ mas pix $^{-1}$ (H2) and $12.251 \pm 0.009$ mas pix $^{-1}(H 3)$ and a North correction angle of $-1.75 \pm 0.08^{\circ}$. The absolute magnitudes were computed using the 2MASS values (Cutri et al. 2003) for the stellar magnitudes.

\section{Orbital analysis}

We retrieved the RV measurements in Bouchy et al. (2016) through the VizieR interface. With only one imaging data point, there is still an ambiguity in the inclination and longitude of the ascending node. To solve for this, we also searched for an astrometric signature of the companion in the HIPPARCosGaia catalog of accelerations (Brandt 2018, 2019): pmra_g_hg $=-2.837 \pm 0.140$ mas $\mathrm{yr}^{-1}$ and pmdec_g_hg $=-0.515 \pm 0.082$ mas $\mathrm{yr}^{-1}$ for Gaia (Gaia Collaboration 2018), pmra_h_hg $=9.411$

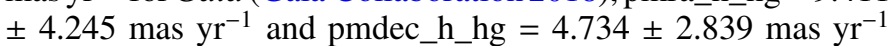
for HIPPARCOS (Perryman et al. 1997; van Leeuwen 2007). These values imply an astrometric detection at $(20.3,6.3) \sigma$ with Gaia and $(2.1,1.7) \sigma$ with HIPPARCOS. We verified that the Gaia DR2 record is well behaved, with a renormalized unit weight error below 1.4 (Lindegren et al. 2018).

We performed a joint fit of the RV, imaging, and proper motion data with the parallel-tempered Markov chain Monte Carlo (MCMC) algorithm provided in the emcee package (Foreman-Mackey et al. 2013), which is based on the algorithm described by Earl \& Deem (2005). Our implementation follows Brandt et al. (2019a) in the broad lines. We sampled the parameter space of our 13-parameter model assuming 15 temperatures for the chains and 100 walkers. The first 8 parameters are the semi-major axis $a$, the eccentricity $e$ and argument of periastron passage $\omega$ (parameterized as $\sqrt{e} \cos \omega$ and $\sqrt{e} \sin \omega$ ), the inclination $i$, the longitude of the ascending node $\Omega$, the time at periastron passage $T_{0}$, the RV semi-amplitude of the star $\kappa_{A}$, and the 
Table 1. Relative photometry and astrometry of HD 72946B.

\begin{tabular}{cccccccc}
\hline \hline Filter & $\begin{array}{c}\lambda_{0} \\
(\mu \mathrm{m})\end{array}$ & $\begin{array}{c}\Delta \lambda \\
(\mu \mathrm{m})\end{array}$ & $\begin{array}{c}\Delta \text { mag } \\
(\mathrm{mag})\end{array}$ & $\begin{array}{c}\text { Abs. mag. } \\
(\mathrm{mag})\end{array}$ & $\begin{array}{c}\text { Flux } \\
\left(\times 10^{-15} \mathrm{~W} \mathrm{~m}^{-2} \mu \mathrm{m}^{-1}\right)\end{array}$ & $\begin{array}{c}\text { Separation } \\
(\mathrm{mas})\end{array}$ & $\begin{array}{c}\text { PA } \\
\left({ }^{\circ}\right)\end{array}$ \\
\hline$H 2$ & 1.593 & 0.052 & $8.97 \pm 0.07$ & $12.51 \pm 0.07$ & $3.027 \pm 0.188$ & $235.7 \pm 2.0$ & $33.65 \pm 0.31$ \\
$H 3$ & 1.667 & 0.054 & $8.81 \pm 0.07$ & $12.35 \pm 0.07$ & $3.236 \pm 0.204$ & $235.6 \pm 2.0$ & $33.68 \pm 0.31$ \\
\hline
\end{tabular}

Notes. The photometric error bars were derived assuming an error budget including the measurement uncertainties (image post-processing) and systematic uncertainties (temporal variability of the flux calibration and of the science sequence).

systemic velocity $\gamma$. We present the results for $\Omega$ and $\omega$ as relative to the companion. To fit the imaging and proper motion data, we used the equations in Appendix A of Makarov \& Kaplan (2005).

The initial state of the sampler was set assuming uniform priors in $\log a, \sqrt{e} \cos \omega, \sqrt{e} \sin \omega, \Omega, T_{0}$, and $\kappa_{A}$, as well as a $\sin i$ prior for $i$. The width of the priors were selected from the results in Bouchy et al. (2016) and a fit to the RV and imaging data with a least-squares Monte Carlo approach (Maire et al. 2015; Schlieder et al. 2016) to derive first ranges for $i$ and $\Omega$. We disentangled the two $(i, \Omega)$ solutions by comparing the predictions for the instantaneous stellar proper motions to the measurements.

The next two parameters in our model are the parallax and the semi-major axis of the orbit of the host star around the center of mass of the system. For the parallax, we drew the initial guesses around the nominal value measured by Gaia assuming a combination of a Gaussian distribution for the measurement uncertainties and a uniform distribution for potential systematics $(<0.1 \mathrm{mas})^{2}$. We drew the semi-major axis of the star around a guess value computed from its mass $\left(0.99 M_{\odot}\right)$, the companion mass $\left(0.07 M_{\odot}\right)$, and the total semi-major axis, assuming a uniform distribution with a half-width of 1.5 mas. The last free model parameters are one RV offset and two RV jitters, using the results in Bouchy et al. (2016) as first guesses.

We ran the MCMC for 125000 iterations and verified the convergence of the chains using the integrated autocorrelation time (Foreman-Mackey et al. 2013; Goodman \& Weare 2010). The posterior distributions in Appendix $C$ were obtained after thinning the chains by a factor 100 to mitigate the correlations and discarding the first $75 \%$ of the chains as the burn-in phase. The median values with $1 \sigma$ uncertainties and the best-fit values of the parameters are given in Table 2. The uncertainties in the parameters in common with Bouchy et al. (2016) are slightly larger or similar. A sample of model orbits is shown in Fig. 2.

We note that the proper motion anomaly measured by HIPPARCOS in RA is different by $\sim 2 \sigma$ from the orbital predictions, whereas the measurement in DEC is well reproduced within the uncertainties. The HIPPARCos and Gaia data affect the derived orbital parameters and dynamical mass within the uncertainties with respect to a fit that only uses the RV and imaging data, except for breaking the ambiguity in the inclination and longitude of ascending node.

\section{Spectral analysis}

We used the IRDIS dual-band photometry of the companion to compute the color-magnitude diagram in Appendix D (details from Appendix C of Bonnefoy et al. 2018). We note that HD 72946B is located near mid-L template dwarfs and is close to HIP 65426b (Chauvin et al. 2017).

2 https://www.cosmos.esa.int/web/gaia/dr2
Table 2. Orbital parameters and dynamical mass of HD 72946B.

\begin{tabular}{|c|c|c|c|}
\hline Parameter & Unit & Median $\pm 1 \sigma$ & Best fit \\
\hline \multicolumn{4}{|c|}{ Fitted parameters } \\
\hline Semi-major axis $a$ & mas & $249.1_{-3.0}^{+3.1}$ & 250.4 \\
\hline$\sqrt{e} \cos \omega$ & & $0.231_{-0.020}^{+0.019}$ & 0.230 \\
\hline$\sqrt{e} \sin \omega$ & & $0.662_{-0.009}^{+0.008}$ & 0.663 \\
\hline Inclination $i$ & $\circ$ & $59.3_{-2.3}^{+2.3}$ & 59.6 \\
\hline PA of asc. node $\Omega$ & $\circ$ & $-12.0_{-3.9}^{+4.3}$ & -11.8 \\
\hline Time periastron $T_{0}$ & BJD & $2455956.7_{-10.1}^{+10.7}$ & 2455955.1 \\
\hline RV semi-ampl. $\kappa_{A}$ & $\mathrm{~ms}^{-1}$ & $778.7_{-9.4}^{+10.5}$ & 774.1 \\
\hline Syst. velocity $\gamma$ & $\mathrm{ms}^{-1}$ & $-203.2_{-86}^{+8.4}$ & -207.4 \\
\hline Parallax $\pi$ & mas & $38.65 \pm 0.12$ & 38.66 \\
\hline SMA primary $a_{1}$ & mas & $16.28_{-0.26}^{+0.29}$ & 16.12 \\
\hline $\mathrm{RV}$ offset $\mathrm{ZP}_{\text {SOPHIE }}$ & $\mathrm{m} \mathrm{s}^{-1}$ & $90.6_{-16.9}^{+15.20}$ & 96.2 \\
\hline RV jitter $\sigma_{\text {ELODIE }}$ & $\mathrm{ms} \mathrm{s}^{-1}$ & $24.4_{-3.3}^{+4.1}$ & 22.9 \\
\hline $\mathrm{RV}$ jitter $\sigma_{\text {SOPHIE }}$ & $\mathrm{ms}^{-1}$ & $16.1_{-3.5}^{+5.0}$ & 12.67 \\
\hline \multicolumn{4}{|c|}{ Computed parameters } \\
\hline$M_{1}$ & $M_{\odot}$ & $0.99 \pm 0.03$ & 1.01 \\
\hline$M_{2}$ & $M_{J}$ & $72.4 \pm 1.6$ & 72.5 \\
\hline Mass ratio $M_{2} / M_{1}$ & & $0.070 \pm 0.002$ & 0.069 \\
\hline Period $P$ & $\mathrm{yr}$ & $15.91_{-0.13}^{+0.16}$ & 15.90 \\
\hline Semi-major axis $a$ & $\mathrm{au}$ & $6.45_{-0.07}^{+0.08}$ & 6.48 \\
\hline Eccentricity $e$ & & $0.493_{-0.008}^{+0.007}$ & 0.493 \\
\hline Arg. periastron $\omega$ & ○ & $250.7 \pm 1.7$ & 250.9 \\
\hline
\end{tabular}

We compared the IFS spectrum to spectra of template dwarfs of the SpeX spectral library using the SPLAT toolkit (Burgasser 2014). Figure 3 shows the reduced $\chi^{2}$ as a function of the spectral type. We include the uncertainties of the template spectra in the $\chi^{2}$ computation. The best-fit object is the red L dwarf 2MASS J03552337+1133437 (Bardalez Gagliuffi et al. 2014) (reduced $\chi^{2}=0.89$, assuming 38 degrees of freedom), which is classified as L5 $\gamma$ by Cruz et al. (2009). From a parabolic fit to the $\chi^{2}$ values, we estimate a spectral type of L5.0 \pm 1.5 considering all spectral types that satisfy $\chi^{2}<\chi_{\min }^{2}+1$.

To fit the spectrophotometry of HD 72946B with atmospheric models, we converted the contrast measurements into physical fluxes using a model spectrum for the star $\left(T_{\text {eff }}=5600 \mathrm{~K}, \log g=4.5 \mathrm{dex}\right.$, and $\left.[\mathrm{Fe} / \mathrm{H}]=0.0 \mathrm{dex}\right)$ from the BT-NextGen library (Allard et al. 2012) and the SPHERE filter transmission curves. The BT-NextGen spectrum is fit to the stellar spectral energy distribution (SED) over the range $0.3-12 \mu \mathrm{m}$ using the Virtual Observatory SED Analyzer (Bayo et al. 2008). The stellar SED is built using data from Tycho ( $\mathrm{H} \varnothing \mathrm{g}$ et al. 2000), 2MASS (Cutri et al. 2003), WISE (Cutri et al. 2013), and IRAS (Helou \& Walker 1988), as well as Johnson photometry (Mermilliod 2006) and Strömgren photometry (Paunzen 2015). 

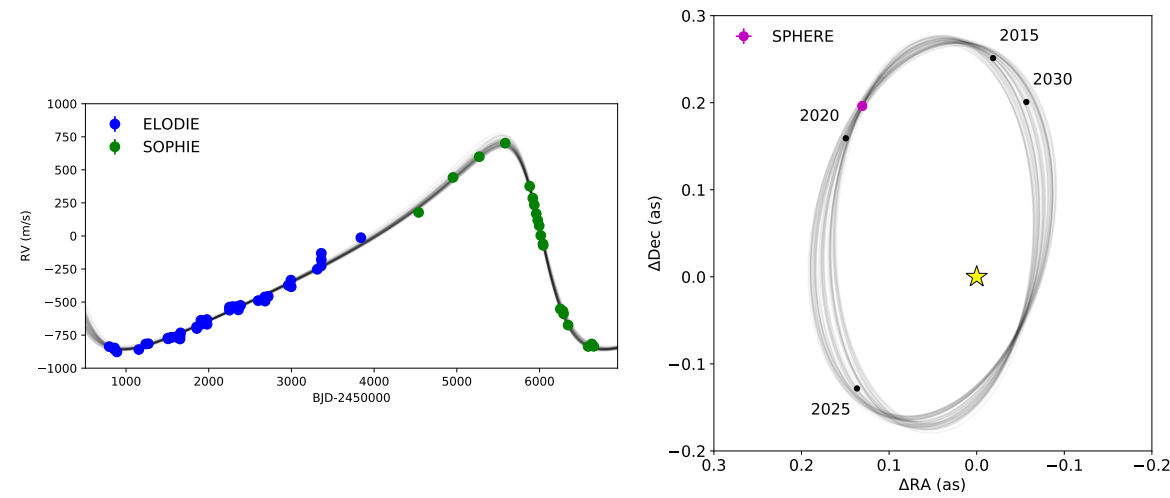

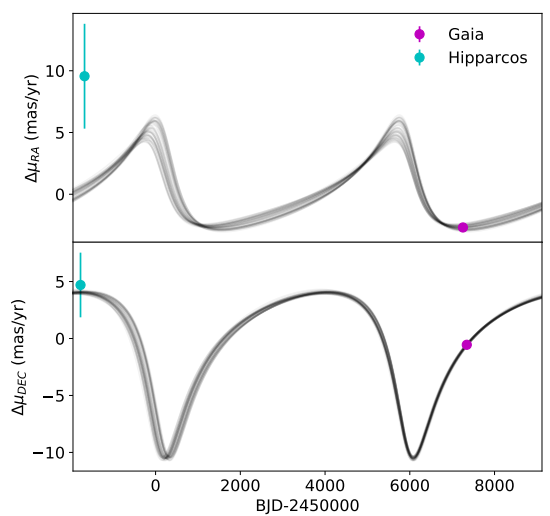

Fig. 2. Sample of 50 model orbits fitted on the HD 72946B data (colored points) from RV (left), imaging (middle), and astrometry (right). Middle panel: yellow star marks the location of the primary star, and the black dots show the median predicted position for given epochs.

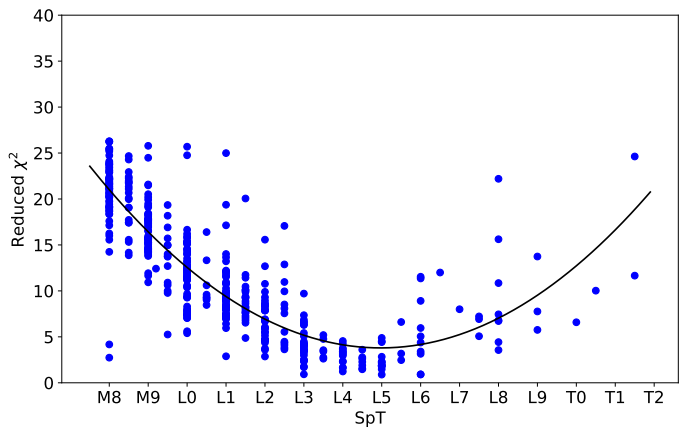

Fig. 3. Reduced $\chi^{2}$ as a function of the spectral type of the comparison of the IFS spectrum of HD 72946B to SpeX template dwarfs.

We show in Fig. 4 the resulting SED of HD 72946B. We performed a grid search for best-fit models in the BT-Settl spectral library (Allard et al. 2011). The characteristics of the grid are $T_{\text {eff }}=700-2500 \mathrm{~K}$ by steps of $100 \mathrm{~K}, \log g=3.5-5.5$ dex by steps of $0.5 \mathrm{dex}$, and $[\mathrm{Fe} / \mathrm{H}]=0.0 \mathrm{dex}$. We allowed the radius to vary and kept solutions with radii in the range $0.7-1.1 R_{J}$. We show the four best-match model spectra in Fig. 4. An effective temperature of $\sim 1700 \mathrm{~K}$ provides a good match to the data, which is in the range expected from evolutionary models for an age of $\sim 1-3$ Gyr given the dynamical mass. It also agrees with a spectral type of L5 from the relation for field dwarfs in Filippazzo et al. (2015; left panel of their Fig. 15).

\section{Discussion}

HD 72946B joins the short list of benchmark brown dwarf companions to stars with RV and imaging measurements: HR 7672B (Liu et al. 2002; Crepp et al. 2012), HD 19467B (Crepp et al. 2014), HD 4747B (Sahlmann et al. 2011; Crepp et al. 2016; Peretti et al. 2019), GJ 758B (Thalmann et al. 2009; Bowler et al. 2018), HD 4113C (Cheetham et al. 2018a), and GJ 229B (Nakajima et al. 1995; Brandt et al. 2019b). HD 72946B stands out among these objects because a complete orbit is covered by $\mathrm{RV}$ and it has the smallest physical separation to the star, $\sim 6.4-6.5 \mathrm{au}$. This is slightly outside the ice line for a Sun-like star.

To evaluate a possible formation mechanism for HD 72946B, we compared its mass (or mass ratio to the star) and separation to model objects formed by fragmentation of a collapsing cloud in Bate (2009) (Fig. 21) or by disk gravitational instabilities in Forgan \& Rice (2013) and Vigan et al. (2017) (left panel

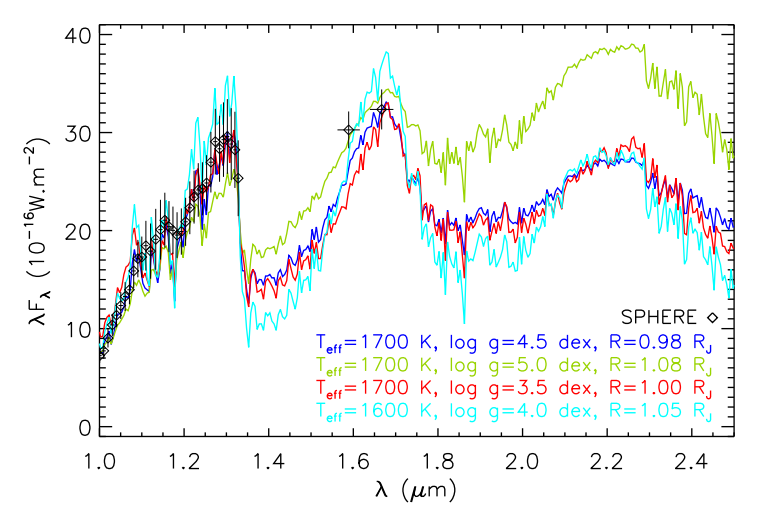

Fig. 4. Spectral energy distribution of HD 72946B (black). The four best-match BT-Settl spectra are shown for comparison (colors).

of Fig. 8 in the latter paper). The semi-major axes of most of the model objects with mass ratios similar to HD 72946B formed in the former process are in the range 20-5000 au. The semimajor axes of most of the model objects with masses similar to HD 72946B formed in the latter process are in the range 10-50 au. This means that the semi-major axis of HD 72946B is smaller than those of model objects from both formation mechanisms, and we cannot exclude any of them.

Figure 5 shows the estimated bolometric luminosity and age of HD 72946B with the predictions from the models COND (Baraffe et al. 2003), Saumon \& Marley (2008; for two treatments of the clouds, hybrid and no clouds), Burrows et al. (1997), and Baraffe et al. (2015) assuming the 95.4\% confidence interval for the companion mass from the orbital fit $\left(72.4 \pm 3.2 M_{J}\right)$. We estimate the bolometric luminosity to be $\log \left(L / L_{\odot}\right)=-4.11 \pm 0.10 \mathrm{dex}$ using the magnitude-bolometric luminosity relation in Filippazzo et al. (2015) for field dwarfs and the $J_{s}$ magnitude computed from the IFS spectrum $(15.44 \pm 0.13 \mathrm{mag})$ with a correction of $0.05 \mathrm{dex}$ between the $J_{s}$ and $J$ bands estimated using SpeX spectra of the three best-fit template dwarfs. The $J-H 3$ color of the companion $(1.08 \pm 0.08 \mathrm{mag})$ is consistent with expectations from mid-L field dwarfs (Cheetham et al. 2019) and is closer to the color predicted given the mass and age of the companion by the DUSTY model (cloudy atmosphere, $J-H 3>1$ mag Chabrier et al. 2000) than to the color predicted by the COND model (cloudless atmosphere, $J-H 3<0.8 \mathrm{mag})^{3}$. This suggests a cloudy atmosphere. For ages younger than $800 \mathrm{Myr}$, HD $72946 \mathrm{~B}$ is fainter than the
3 Tables available at http://perso.ens-lyon.fr/france. allard/ 


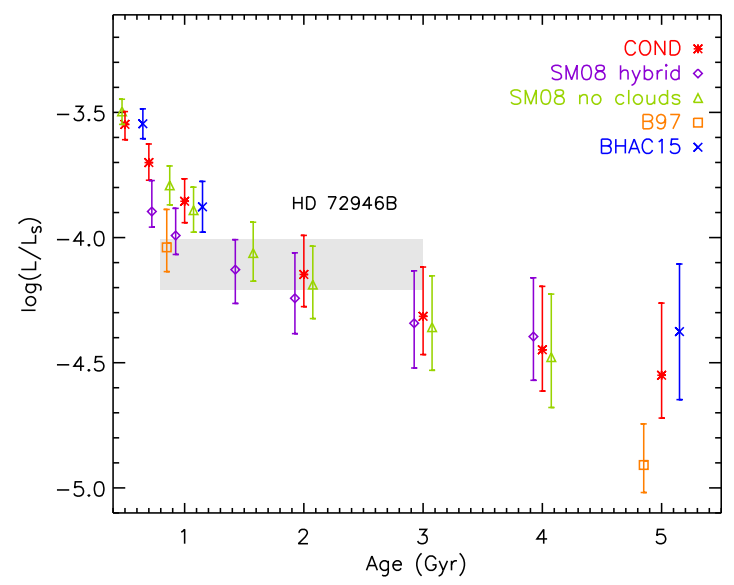

Fig. 5. Bolometric luminosity vs. age of HD 72946B (gray area) compared to evolutionary tracks from the models COND (Baraffe et al. 2003), Saumon \& Marley (2008; for two treatments of the clouds), Burrows et al. (1997), and Baraffe et al. (2015) assuming the mass range for the companion from the orbital fit (data points). Small horizontal offsets are applied to all models except for COND for clarity.

predictions of all evolutionary models. At $1 \mathrm{Gyr}$, the companion properties are best reproduced by the hybrid cloud model of Saumon \& Marley (2008) and Burrows et al. (1997). At 2 Gyr, the best-match models are COND and the cloudless model of Saumon \& Marley (2008), and Baraffe et al. (2015). At 3 Gyr, the models of Baraffe et al. (2015) account better for the companion properties. Observations to better constrain the stellar age with gyrochronology may allow a better distinction between the models.

The characterization of HD 72946B clearly illustrates the improvements in the high-contrast imaging instrumentation toward bridging the gap in separation to the star with RV and astrometry. The combination of these data provides stronger constraints on the properties of substellar companions than can be reached with one technique alone. This allows testing their massluminosity models. The SPHERE data are sensitive to low-mass brown dwarfs down to $\sim 30 M_{J}$ at separations as close as $0.2^{\prime \prime}$ (Appendix E). The next generation of high-contrast imaging instruments on extremely large telescopes will enable extending analyses like this to the bulk of substellar companions that are detected with RV at closer separations and at lower masses down to the planetary regime and building empirical mass-luminosity relations for exoplanets. The future release of the Gaia epoch astrometry will permit more accurate measurements of proper motion anomalies. This will improve dynamical mass estimates and provide new targets for this purpose.

Acknowledgements. The authors thank the referee for a constructive report that helped to clarify the manuscript. The authors thank the ESO Paranal Staff for support in conducting the observations. N. G. and J. O. acknowledge financial support from the ICM (Iniciativa Científica Milenio) via the Núcleo Milenio de Formación Planetaria grant, from the Universidad de Valparaíso, and from Fondecyt (grant 1180395). N. G. acknowledges grant support from project CONICYT-PFCHA/Doctorado Nacional/2017 folio 21170650 We acknowledge financial support from the Programme National de Planétologie (PNP), the Programme National de Physique Stellaire (PNPS) of CNRSINSU in France, and the Agence Nationale de la Recherche (GIPSE project; grant ANR-14-CE33-0018). T. H. acknowledges support from the European Research Council under the Horizon 2020 Framework Program via the ERC Advanced Grant Origins 8324 28. This work made use of recipes from the IDL libraries: IDL Astronomy Users's Library (Landsman 1993), Coyote (http://www.idlcoyote.com/index.html), EXOFAST (Eastman et al 2013), JBIU (http://www.simulated-galaxies.ua.edu/jbiu/), and the s3drs package (http://www.heliodocs.com/xdoc/index.html). It also made use of the Python packages: NumPy (Oliphant 2006), emcee (Foreman-Mackey et al. 2013), corner (Foreman-Mackey 2016), Matplotlib (Hunter 2007), Astropy (Astropy Collaboration 2013, 2018), and dateutil (http: //dateutil.readthedocs.io/). This publication makes use of VOSA, developed under the Spanish Virtual Observatory project supported by the Spanish MINECO through grant AyA2017-84089. VOSA has been partially updated by using funding from the European Union's Horizon 2020 Research and Innovation Programme, under Grant Agreement $n^{\circ} 776403$ (EXOPLANETS-A). This research has benefitted from the SpeX Prism Spectral Libraries, maintained by Adam Burgasser at http://pono.ucsd.edu/ adam/ browndwarfs/spexprism. This research made use of the SIMBAD database and the VizieR Catalogue access tool, both operated at the CDS, Strasbourg, France. The original descriptions of the SIMBAD and VizieR services were published in Wenger et al. (2000) and Ochsenbein et al. (2000). This research has made use of NASA's Astrophysics Data System Bibliographic Services.

\section{References}

Aguilera-Gómez, C., Ramírez, I., \& Chanamé, J. 2018, A\&A, 614, A55

Allard, F., Homeier, D., \& Freytag, B. 2011, in Astronomical Society of the Pacific (ASP) Conference Series, eds. C. Johns-Krull, M. K. Browning, \& A. A. West, 448, 91

Allard, F., Homeier, D., Freytag, B., \& Sharp, C. M. 2012, in EAS Publications Series, eds. C. Reylé, C. Charbonnel, \& M. Schultheis, 57, 3

Astropy Collaboration (Robitaille, T. P., et al.) 2013, A\&A, 558, A33

Astropy Collaboration (Price-Whelan, A. M., et al.) 2018, AJ, 156, 123

Bailey, V., Meshkat, T., Reiter, M., et al. 2014, ApJ, 780, L4

Baraffe, I., Chabrier, G., Barman, T. S., Allard, F., \& Hauschildt, P. H. 2003, A\&A, 402, 701

Baraffe, I., Homeier, D., Allard, F., \& Chabrier, G. 2015, A\&A, 577, A42

Bardalez Gagliuffi, D. C., Burgasser, A. J., Gelino, C. R., et al. 2014, ApJ, 794, 143

Bate, M. R. 2009, MNRAS, 392, 590

Bayo, A., Rodrigo, C., Barrado Y Navascués, D., et al. 2008, A\&A, 492, 277

Beichman, C., Gelino, C. R., Kirkpatrick, J. D., et al. 2014, ApJ, 783, 68

Beuzit, J. L., Vigan, A., Mouillet, D., et al. 2019, A\&A, 631, A155

Boccaletti, A., Sezestre, E., Lagrange, A.-M., et al. 2018, A\&A, 614, A52

Bonnefoy, M., Chauvin, G., Lagrange, A.-M., et al. 2014, A\&A, 562, A127

Bonnefoy, M., Perraut, K., Lagrange, A. M., et al. 2018, A\&A, 618, A63

Boro Saikia, S., Marvin, C. J., Jeffers, S. V., et al. 2018, A\&A, 616, A108

Boss, A. P. 1997, Science, 276, 1836

Bouchy, F., Ségransan, D., Díaz, R. F., et al. 2016, A\&A, 585, A46

Bowler, B. P., Dupuy, T. J., Endl, M., et al. 2018, AJ, 155, 159

Brandt, T. D. 2018, ApJS, 239, 31

Brandt, T. D. 2019, ApJS, 241, 39

Brandt, T. D., Dupuy, T. J., \& Bowler, B. P. 2019a, AJ, 158, 140

Brandt, T. D., Dupuy, T. J., Bowler, B. P., et al. 2019b, AJ, submitted [arXiv:1910.01652]

Burgasser, A. J. 2014, Astron. Soc. India Conf. Ser., 11, 7

Burningham, B., Pinfield, D. J., Leggett, S. K., et al. 2008, MNRAS, 391, 320

Burrows, A., Marley, M., Hubbard, W. B., et al. 1997, ApJ, 491, 856

Cantalloube, F., Mouillet, D., Mugnier, L. M., et al. 2015, A\&A, 582, A89

Carbillet, M., Bendjoya, P., Abe, L., et al. 2011, Exp. Astron., 30, 39

Casagrande, L., Schönrich, R., Asplund, M., et al. 2011, A\&A, 530, A138

Chabrier, G., Baraffe, I., Allard, F., \& Hauschildt, P. 2000, ApJ, 542, 464

Chauvin, G., Desidera, S., Lagrange, A.-M., et al. 2017, A\&A, 605, L9

Cheetham, A., Ségransan, D., Peretti, S., et al. 2018a, A\&A, 614, A16

Cheetham, A., Bonnefoy, M., Desidera, S., et al. 2018b, A\&A, 615, A160

Cheetham, A. C., Samland, M., Brems, S. S., et al. 2019, A\&A, 622, A80

Claudi, R. U., Turatto, M., Gratton, R. G., et al. 2008, SPIE Conf. Ser., 7014, $70143 \mathrm{E}$

Crepp, J. R., Johnson, J. A., Fischer, D. A., et al. 2012, ApJ, 751, 97

Crepp, J. R., Johnson, J. A., Howard, A. W., et al. 2014, ApJ, 781, 29

Crepp, J. R., Gonzales, E. J., Bechter, E. B., et al. 2016, ApJ, 831, 136

Cruz, K. L., Kirkpatrick, J. D., \& Burgasser, A. J. 2009, AJ, 137, 3345

Cutri, R. M., Skrutskie, M. F., van Dyk, S., et al. 2003, VizieR Online Data Catalog: II/246

Cutri, R. M., Wright, E. L., Conrow, T., et al. 2013, VizieR Online Data Catalog: II/328

da Silva, L., Girardi, L., Pasquini, L., et al. 2006, A\&A, 458, 609

De Rosa, R. J., Patience, J., Ward-Duong, K., et al. 2014, MNRAS, 445, 3694

Delorme, P., Delfosse, X., Albert, L., et al. 2008, A\&A, 482, 961

Delorme, P., Schmidt, T., Bonnefoy, M., et al. 2017, A\&A, 608, A79

Dieterich, S. B., Weinberger, A. J., Boss, A. P., et al. 2018, ApJ, 865, 28

Dohlen, K., Langlois, M., Saisse, M., et al. 2008, SPIE Conf. Ser., 7014, 70143L

Dommanget, J., \& Nys, O. 2002, VizieR Online Data Catalog: I/274 
Ducourant, C., Teixeira, R., Galli, P. A. B., et al. 2014, A\&A, 563, A121 Dupuy, T. J., \& Kraus, A. L. 2013, Science, 341, 1492

Dupuy, T. J., \& Liu, M. C. 2017, ApJS, 231, 15

Duquennoy, A., \& Mayor, M. 1991, A\&A, 500, 337

Earl, D. J., \& Deem, M. W. 2005, Phys. Chem. Chem. Phys., 7, 3910

Eastman, J., Gaudi, B. S., \& Agol, E. 2013, PASP, 125, 83

Faherty, J. K., Burgasser, A. J., Walter, F. M., et al. 2012, ApJ, 752, 56

Feroz, F., Balan, S. T., \& Hobson, M. P. 2011, MNRAS, 416, L104

Filippazzo, J. C., Rice, E. L., Faherty, J., et al. 2015, ApJ, 810, 158

Foreman-Mackey, D. 2016, J. Open Source Softw., 1, 24

Foreman-Mackey, D., Hogg, D. W., Lang, D., \& Goodman, J. 2013, PASP, 125, 306

Forgan, D., \& Rice, K. 2013, MNRAS, 432, 3168

Gaia Collaboration (Prusti, T., et al.) 2016, A\&A, 595, A1

Gaia Collaboration (Brown, A. G. A., et al.) 2018, A\&A, 616, A1

Galicher, R., Boccaletti, A., Mesa, D., et al. 2018, A\&A, 615, A92

Gauza, B., Béjar, V. J. S., Pérez-Garrido, A., et al. 2015, ApJ, 804, 96

Gizis, J. E., Allers, K. N., Liu, M. C., et al. 2015, ApJ, 799, 203

Goodman, J., \& Weare, J. 2010, Appl. Math. Comput. Sci., 5, 65

Gray, R. O., Corbally, C. J., Garrison, R. F., McFadden, M. T., \& Robinson, P. E. 2003, AJ, 126, 2048

Grether, D., \& Lineweaver, C. H. 2006, ApJ, 640, 1051

Hayes, D. S. 1985, in Calibration of Fundamental Stellar Quantities, eds. D. S. Hayes, L. E. Pasinetti, \& A. G. D. Philip, IAU Symp., 111, 225

Helou, G., \& Walker, D. W. 1988, Infrared Astronomical Satellite (IRAS) Catalogs and Atlases. Volume 7: The Small Scale Structure Catalog, 7, 1

Høg, E., Fabricius, C., Makarov, V. V., et al. 2000, A\&A, 355, L27

Hunter, J. D. 2007, Comput. Sci. Eng., 9, 90

Kirkpatrick, J. D., Reid, I. N., Liebert, J., et al. 2000, AJ, 120, 447

Kirkpatrick, J. D., Cushing, M. C., Gelino, C. R., et al. 2011, ApJS, 197, 19

Kirkpatrick, J. D., Gelino, C. R., Cushing, M. C., et al. 2012, ApJ, 753, 156

Konopacky, Q. M., Ghez, A. M., Barman, T. S., et al. 2010, ApJ, 711, 1087

Konopacky, Q. M., Thomas, S. J., Macintosh, B. A., et al. 2014, in Groundbased and Airborne Instrumentation for Astronomy V, SPIE Conf. Ser., 9147 914784

Lachapelle, F.-R., Lafrenière, D., Gagné, J., et al. 2015, ApJ, 802, 61

Lafrenière, D., Jayawardhana, R., \& van Kerkwijk, M. H. 2010, ApJ, 719, 497

Landsman, W. B. 1993, in Astronomical Data Analysis Software and Systems II, eds. R. J. Hanisch, R. J. V. Brissenden, \& J. Barnes, ASP Conf. Ser., 52, 246

Langlois, M., Vigan, A., Moutou, C., et al. 2013, in Proceedings of the Third AO4ELT Conference, eds. S. Esposito, \& L. Fini, 63

Leggett, S. K., Allard, F., Dahn, C., et al. 2000, ApJ, 535, 965

Lindegren, L., Hernández, J., Bombrun, A., et al. 2018, A\&A, 616, A2

Liu, M. C., Fischer, D. A., Graham, J. R., et al. 2002, ApJ, 571, 519

Liu, M. C., Magnier, E. A., Deacon, N. R., et al. 2013, ApJ, 777, L20

Liu, M. C., Dupuy, T. J., \& Allers, K. N. 2016, ApJ, 833, 96

Lucas, P. W., Tinney, C. G., Burningham, B., et al. 2010, MNRAS, 408, L56

Luck, R. E. 2017, AJ, 153, 21

Luck, R. E., \& Heiter, U. 2006, AJ, 131, 3069

Luhman, K. L., \& Esplin, T. L. 2016, AJ, 152, 78

Ma, B., \& Ge, J. 2014, MNRAS, 439, 2781

Mace, G. N., Kirkpatrick, J. D., Cushing, M. C., et al. 2013, ApJS, 205, 6

Macintosh, B., Graham, J. R., Ingraham, P., et al. 2014, PNAS, 111, 12661

Maire, A.-L., Skemer, A. J., Hinz, P. M., et al. 2015, A\&A, 576, A133

Maire, A.-L., Langlois, M., Dohlen, K., et al. 2016, SPIE Conf. Ser., 9908 990834

Makarov, V. V., \& Kaplan, G. H. 2005, AJ, 129, 2420

Mamajek, E. E., \& Hillenbrand, L. A. 2008, ApJ, 687, 1264

Marois, C., Lafrenière, D., Doyon, R., Macintosh, B., \& Nadeau, D. 2006, ApJ, 641, 556

Marois, C., Correia, C., Galicher, R., et al. 2014, SPIE Conf. Ser., 9148, 91480U

Martinez, P., Dorrer, C., Aller Carpentier, E., et al. 2009, A\&A, 495, 363

Martínez-Arnáiz, R., Maldonado, J., Montes, D., Eiroa, C., \& Montesinos, B. 2010, A\&A, 520, A79

Mawet, D., Milli, J., Wahhaj, Z., et al. 2014, ApJ, 792, 97

Mermilliod, J. C. 2006, VizieR Online Data Catalog: II/168

Mesa, D., Gratton, R., Zurlo, A., et al. 2015, A\&A, 576, A121

Montes, D., López-Santiago, J., Gálvez, M. C., et al. 2001, MNRAS, 328, 45

Mountain, C. M., Leggett, S. K., Selby, M. J., Blackwell, D. E., \& Petford, A. D. 1985, A\&A, 151, 399
Mugnier, L. M., Cornia, A., Sauvage, J.-F., et al. 2009, J. Opt. Soc. Am. A, 26, 1326

Nakajima, T., Oppenheimer, B. R., Kulkarni, S. R., et al. 1995, Nature, 378, 463 Nielsen, E. L., De Rosa, R. J., Macintosh, B., et al. 2019, AJ, 158, 13

Ochsenbein, F., Bauer, P., \& Marcout, J. 2000, A\&AS, 143, 23

Oh, S., Price-Whelan, A. M., Hogg, D. W., Morton, T. D., \& Spergel, D. N. 2017 , AJ, 153, 257

Oliphant, T. E. 2006, A Guide to NumPy (USA: Trelgol Publishing), 1

Patience, J., King, R. R., de Rosa, R. J., \& Marois, C. 2010, A\&A, 517, A76

Paunzen, E. 2015, A\&A, 580, A23

Pavlov, A., Möller-Nilsson, O., Feldt, M., et al. 2008, SPIE Conf. Ser., 7019, 701939

Peretti, S., Ségransan, D., Lavie, B., et al. 2019, A\&A, 631, A107

Perrin, M. D., Maire, J., Ingraham, P., et al. 2014, SPIE Conf. Ser., 9147, 3

Perrin, M. D., Ingraham, P., Follette, K. B., et al. 2016, in Ground-based and Airborne Instrumentation for Astronomy VI, SPIE Conf. Ser., 9908, 990837 Perryman, M. A. C., Lindegren, L., Kovalevsky, J., et al. 1997, A\&A, 500, 501 Pourbaix, D., Tokovinin, A. A., Batten, A. H., et al. 2004, A\&A, 424, 727

Rajan, A., Rameau, J., De Rosa, R. J., et al. 2017, AJ, 154, 10

Ramírez, I., Fish, J. R., Lambert, D. L., \& Allende Prieto, C. 2012, ApJ, 756, 46 Rocha-Pinto, H. J., Flynn, C., Scalo, J., et al. 2004, A\&A, 423, 517

Sahlmann, J., Ségransan, D., Queloz, D., et al. 2011, A\&A, 525, A95

Saumon, D., \& Marley, M. S. 2008, ApJ, 689, 1327

Schneider, A. C., Cushing, M. C., Kirkpatrick, J. D., et al. 2015, ApJ, 804, 92

Schlieder, J. E., Skemer, A. J., Maire, A.-L., et al. 2016, ApJ, 818, 1

Sestito, P., Randich, S., \& Pallavicini, R. 2004, A\&A, 426, 809

Soummer, R., Pueyo, L., \& Larkin, J. 2012, ApJ, 755, L28

Stone, J. M., Skemer, A. J., Kratter, K. M., et al. 2016, ApJ, 818, L12

Thalmann, C., Carson, J., Janson, M., et al. 2009, ApJ, 707, L123

Tinney, C. G., Faherty, J. K., Kirkpatrick, J. D., et al. 2014, ApJ, 796, 39

van Leeuwen, F. 2007, A\&A, 474, 653

Vigan, A., Moutou, C., Langlois, M., et al. 2010, MNRAS, 407, 71

Vigan, A., Gry, C., Salter, G., et al. 2015, MNRAS, 454, 129

Vigan, A., Bonavita, M., Biller, B., et al. 2017, A\&A, 603, A3

Wahhaj, Z., Liu, M. C., Biller, B. A., et al. 2011, ApJ, 729, 139

Warren, S. J., Mortlock, D. J., Leggett, S. K., et al. 2007, MNRAS, 381, 1400

Wenger, M., Ochsenbein, F., Egret, D., et al. 2000, A\&AS, 143, 9

Zapatero Osorio, M. R., Béjar, V. J. S., Miles-Páez, P. A., et al. 2014, A\&A, 568, A6

1 STAR Institute, Université de Liège, Allée du Six Août 19c, 4000 Liège, Belgium e-mail: almaire@uliege.be

2 Department of Physics, University of Oxford, Oxford, UK

3 INAF-Osservatorio Astronomico di Padova, Vicolo dell'Osservatorio 5, 35122 Padova, Italy

4 INAF Catania Astrophysical Observatory, Via S. Sofia 78, 95123 Catania, Italy

5 Max-Planck-Institut für Astronomie, Königstuhl 17, 69117 Heidelberg, Germany

6 Instituto de Física y Astronomía, Facultad de Ciencias, Univ. de Valparaíso, Av. Gran Bretaña 1111, Playa Ancha, Valparaíso, Chile

7 Núcleo Milenio Formación Planetaria - NPF, Univ. de Valparaíso, Av. Gran Bretaña 1111, Playa Ancha, Valparaíso, Chile

8 LESIA, Observatoire de Paris, PSL Research University, CNRS, Sorbonne Universités, UPMC Univ. Paris 06, Univ. Paris Diderot, Sorbonne Paris Cité, 5 Place Jules Janssen, 92195 Meudon, France

9 Univ. Grenoble Alpes, CNRS, IPAG, 38000 Grenoble, France

10 Geneva Observatory, University of Geneva, Chemin des Maillettes 51, 1290 Versoix, Switzerland

11 Unidad Mixta Internacional Franco-Chilena de Astronomía CNRS/INSU UMI 3386 and Departamento de Astronomía, Universidad de Chile, Casilla 36-D, Santiago, Chile

12 CRAL, UMR 5574, CNRS/ENS-Lyon/Université Lyon 1, 9 Av. Ch. André, 69561 Saint-Genis-Laval, France 


\section{Appendix A: Stellar multiplicity}

Bouchy et al. (2016) noted that HD 72946 is part of a multiple system. HD 72945 is a comoving F8 star located at a projected separation of 230 au. Duquennoy \& Mayor (1991) identified it as a spectroscopic binary SB1 with a period of $14.3 \mathrm{~d}$.

The Gaia parallaxes of HD 72945 and HD 72946 differ at the $4.2 \sigma$ level, indicating a distance difference along the line of sight of about $0.41 \pm 0.10 \mathrm{pc}$. The similar proper motion and systematic velocities of the two stars argue in favor of a physical association (see also Oh et al. 2017). We speculate that the separation along the line of sight could be significantly larger than the one projected on the plane of the sky. Alternatively, the parallaxes of one or both components can be altered above the formal errors by the presence of the companions.

In addition, Dommanget \& Nys (2002) reported three stellar companions with angular separations of 93", 117", and 122".

Using the Gaia parallaxes, we find that the three components identified by Dommanget \& Nys (2002) $(\pi<12$ mas) do not form a system with HD72945 and HD72946 $(\pi \sim$ 38-39 mas). Instead, we note in the Gaia catalog a star (2MASS ID 08354678+0635294) at $\sim 130^{\prime \prime}$ ( 3400 au) from HD 72946 with a parallax of $38.8196 \pm 0.0584$ mas (distance along the line of sight $\sim 0.11 \pm 0.05 \mathrm{pc}$ ) and similar proper motion, but without a measured RV. Therefore we argue that the system is formed by four stellar and one substellar components. For the orbital analysis, we assumed that the acceleration seen in the proper motion of HD 72946 is entirely due to the substellar companion HD 72946B.

The other components may provide additional constraints on the age of the system. In particular, we derive for HD 72945 an age and a mass using the PARAM web interface, the $T_{\text {eff }}$ derived by Casagrande et al. (2011) from Strömgren photometry, the Gaia parallax, the $V$ magnitude from HIPPARcos $(5.92 \pm 0.01 \mathrm{mag})$, and the metallicity measured by Bouchy et al. (2016) for HD 72946 (0.01 dex difference only with the metallicity of HD 72945 measured by Casagrande et al. 2011). We find an age of $1.584 \pm 0.952 \mathrm{Gyr}$ and a mass of $1.245 \pm 0.030 M_{\odot}$. For this computation, we assumed that the spectroscopic companion of HD 72945 does not contribute significantly to the integrated photometry. This should be the case if its mass is not much higher than the expected minimum mass from the RV orbit. Using the SB9 orbit (Pourbaix et al. 2004) and the isochronal mass above, we derive a minimum mass for the secondary of $0.34 M_{\odot}$. In any case, a significant contribution to the photometry would shift the measured isochronal age toward higher values than expected. We report archival GPI data of HD 72945 in Appendix F.

\section{Appendix B: Comparison of extracted spectrophotometry}

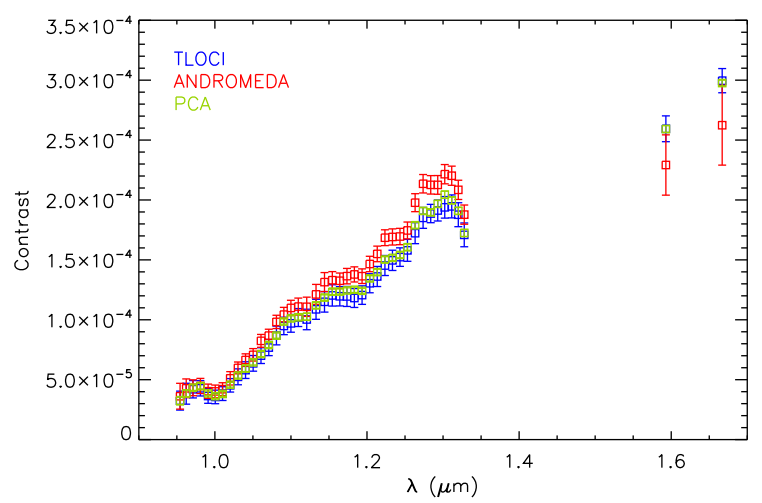

Fig. B.1. Comparison of the TLOCI, ANDROMEDA, and PCA spectrophotometry. The measurement uncertainties are shown at $3 \sigma$ for all algorithms.

We show in Fig. B.1 the comparison of the spectrophotometry extracted with the ANgular DiffeRential Optimal Method Exoplanet Detection Algorithm (ANDROMEDA, Mugnier et al. 2009; Cantalloube et al. 2015) and with the Template Locally Optimized Combination of Images (TLOCI, Marois et al. 2014) and Principal Component Analysis (PCA, Soummer et al. 2012) algorithms provided by the SpeCal pipeline (Galicher et al. 2018). For the TLOCI extraction, we used the fitting of a model planet image, whereas for PCA we employed the negative synthetic planet injection. The fitting uncertainties are given at $3 \sigma$. We note the good agreement between the TLOCI and PCA results within the TLOCI uncertainties. The IFS spectra between TLOCI and ANDROMEDA did not formerly agree for wavelengths longer than $\sim 1.2 \mu \mathrm{m}$, with the ANDROMEDA spectrum showing a steeper slope than the TLOCI spectrum. The IRDIS photometry for ANDROMEDA looks fainter than the TLOCI photometry by $\sim 15 \%$, although the uncertainties are large. This results in an IRDIFS spectrum that is redder for TLOCI than for ANDROMEDA.

We tested both ANDROMEDA and TLOCI SED for the atmospheric fitting. We experienced convergence problems when fitting the ANDROMEDA SED, and we chose the TLOCI SED for the analysis shown in this Letter. We did not notice any significant discrepancies in the extracted astrometry, but we chose to use the TLOCI astrometry for consistency. 


\section{Appendix C: Corner plot of the orbital fit}

We provide here the corner plot of the orbital parameters derived in Sect. 4.

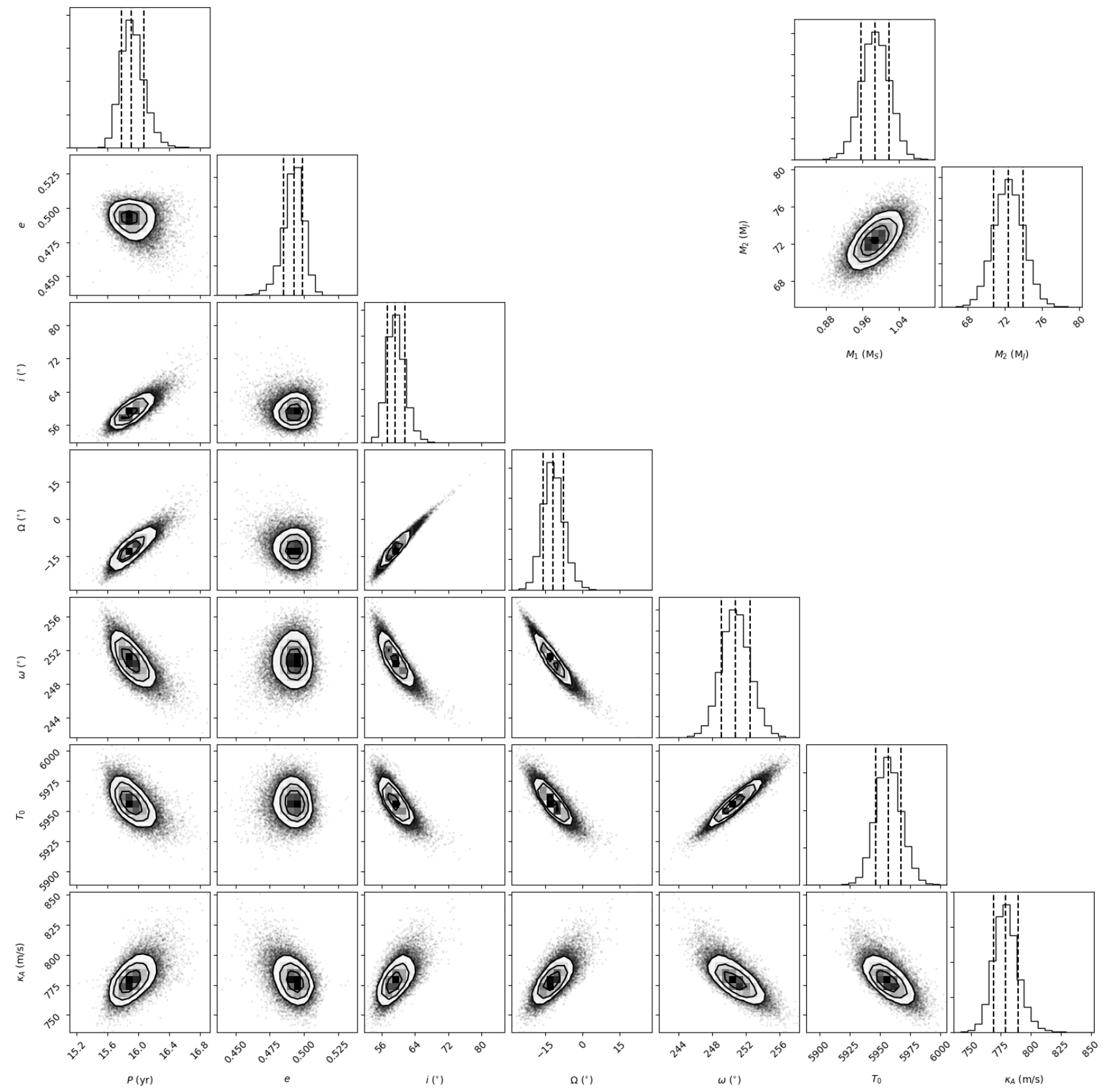

Fig. C.1. MCMC posteriors of the orbital parameters (left) and of the masses of HD 72946 A and B (top right). The diagrams displayed on the diagonals represent the 1D histogram distributions for the parameters. The off-diagonal diagrams show the correlations. In the histograms, the dashed vertical lines indicate the $16 \%, 50 \%$, and $84 \%$ quantiles. 


\section{Appendix D: Color-magnitude diagram}

To build the diagram in Fig. D.1, we used spectra of M, L, and T dwarfs from the SpeX-Prism library (Burgasser 2014) and from Leggett et al. (2000) and Schneider et al. (2015) to generate synthetic photometry in the SPHERE filter passbands. The zero-points were computed using a flux-calibrated spectrum of Vega (Hayes 1985; Mountain et al. 1985). We also considered the spectra of young and/or dusty freefloating objects from Liu et al. (2013), Mace et al. (2013), Gizis et al. (2015), and of young companions (Wahhaj et al. 2011; Gauza et al. 2015; Stone et al. 2016; De Rosa et al. 2014; Lachapelle et al. 2015; Bailey et al. 2014; Rajan et al. 2017; Bonnefoy et al. 2014; Patience et al. 2010; Lafrenière et al. 2010; Chauvin et al. 2017; Delorme et al. 2017; Cheetham et al. 2018b). The colors and absolute fluxes of the benchmark companions and isolated T-type objects were generated from the distance and spectra of these objects in Appendix B in Bonnefoy et al. (2018). To conclude, we used the spectra of Y dwarfs published in Schneider et al. (2015), Warren et al. (2007), Delorme et al. (2008), Burningham et al. (2008), Lucas et al. (2010), Kirkpatrick et al. (2012), and Mace et al. (2013) to extend the diagrams in the late-T and early$\mathrm{Y}$ dwarf domain. We used the distances of the field dwarfs reported in Kirkpatrick et al. (2000), Faherty et al. (2012), Dupuy \& Kraus (2013), Tinney et al. (2014), Beichman et al. (2014), and Luhman \& Esplin (2016). We considered those reported in Kirkpatrick et al. (2011), Faherty et al. (2012), Zapatero Osorio et al. (2014), and Liu et al. (2016) for the dusty dwarfs. The companion distances were taken from van Leeuwen (2007) and Ducourant et al. (2014).

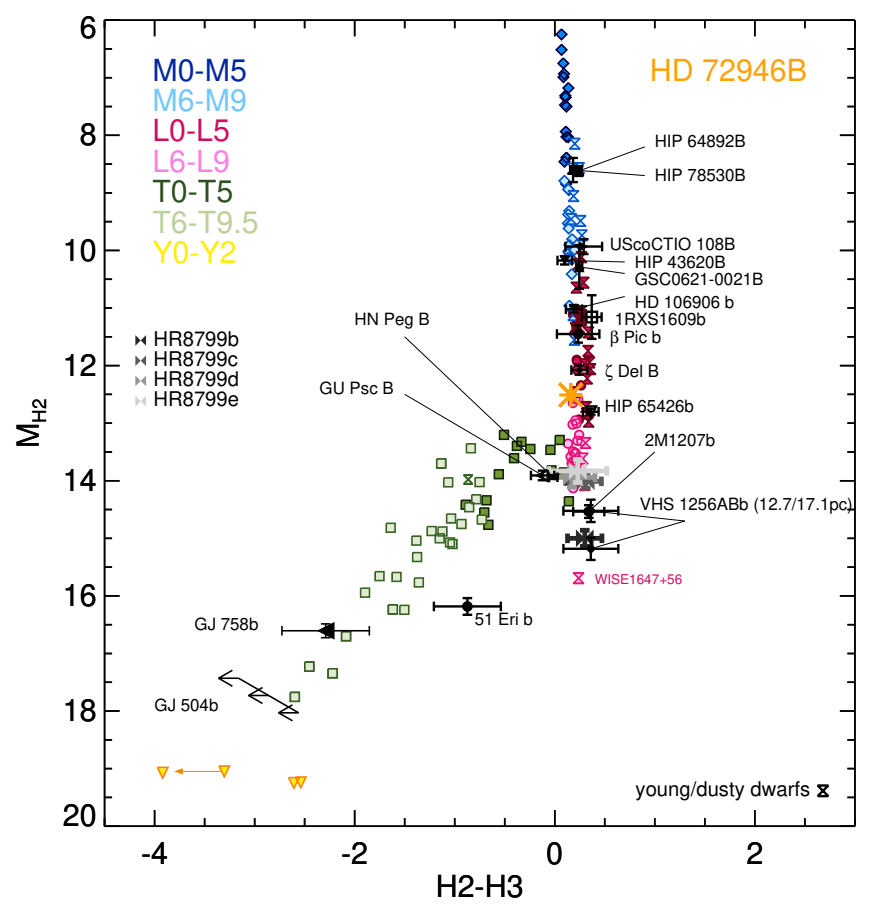

Fig. D.1. Color-magnitude diagram of HD 72946B (orange) using the IRDIS photometry. Template dwarfs (colored points) and a few young low-mass companions (black labels) are also shown for comparison.

\section{Appendix E: SPHERE detection limits}

Figure E. 1 shows the SPHERE detection limits in contrast to the star and the planet mass obtained with ANDROMEDA. For the IFS detection limits, we assumed a T5 dwarf template spectrum (Cantalloube et al., in prep.) retrieved from the SpeX library. The detection limits account for the coronagraphic transmission (Boccaletti et al. 2018) and the small sample statistics correction (Mawet et al. 2014). The contrast to planet mass conversion was derived assuming the "hot-start" evolutionary and atmospheric models of Baraffe et al. (2003, 2015) and an age of 2 Gyr for the system (table from Vigan et al. 2015). The H3 curve is sensitive to more massive objects than the $\mathrm{H} 2$ curve because the probed mass regime corresponds to cold objects with strong methane absorption features and the $\mathrm{H} 3$ filter matches a strong methane band. We excluded additional brown dwarf companions more massive than $\sim 20 M_{J}$ at separations beyond $8 \mathrm{au}$.
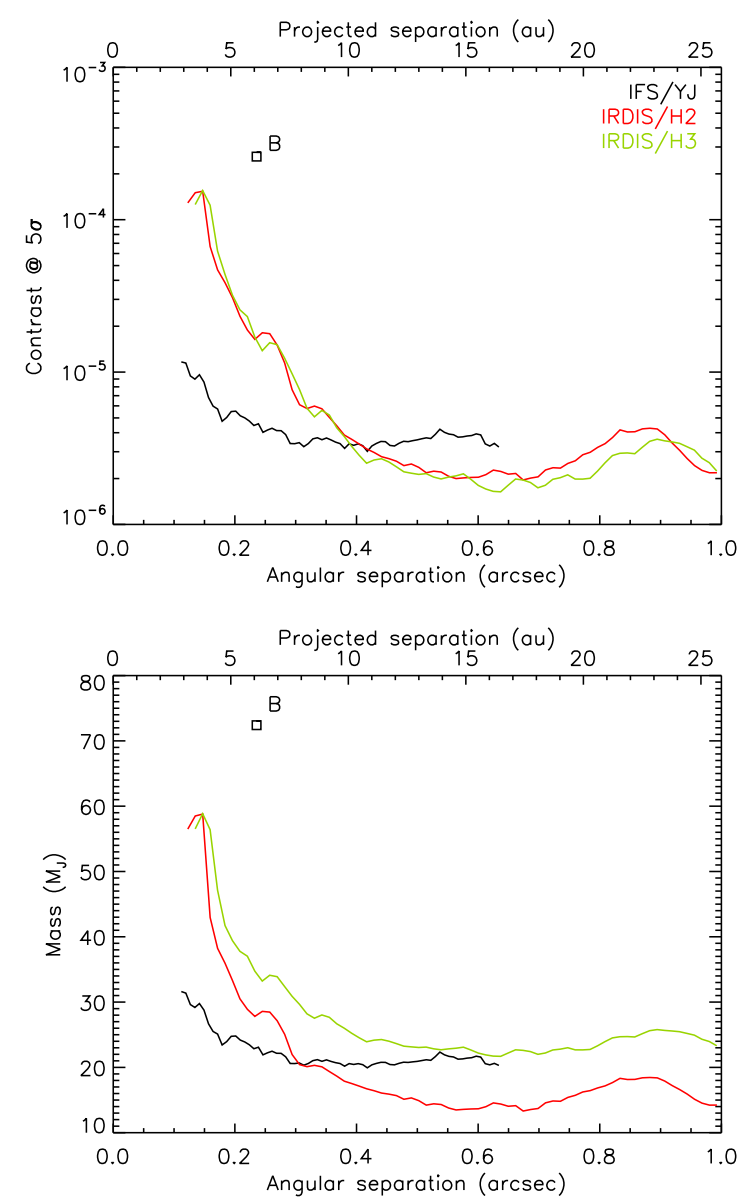

Fig. E.1. SPHERE $5 \sigma$ detection limits in contrast with respect to the star (top) and the planet mass (bottom). We also indicate the location of HD 72946B for comparison. 


\section{Appendix F: GPI archival data of HD 72945}

The stellar SB companion HD 72945 was observed with the Gemini Planet Imager (GPI, Macintosh et al. 2014) on 2015 April 4 UT in the $H$ band. The data were presented in the first statistical analysis of the GPIES survey (Nielsen et al. 2019, target name: HR 3395). The target was observed for an integration time of $32.8 \mathrm{~min}$, which amounts to a field rotation of $19.5^{\circ}$.

We retrieved the data from the Gemini archive and reduced them with the GPI data reduction pipeline v1.4.0 (Perrin et al. 2014, 2016), which applies an automatic correction for the North offset of $-1.00 \pm 0.03^{\circ}$ measured by Konopacky et al. (2014). Then, we post-processed them using ANDROMEDA. No point source is detected above $5 \sigma$. We show in Fig. F.1 the detection limits obtained for a T5 dwarf template spectrum. We assumed an age of 2 Gyr, a distance of 26.3 pc from the Gaia DR2 parallax, and the models of Baraffe et al. (2003, 2015). For the stellar magnitude, we used the 2MASS value, although we note that it is affected by saturation. We included the small sample statistics correction. We cut the curves to separation larger than $0.15^{\prime \prime}$ because we were unable to find GPI coronagraphic transmission curves.
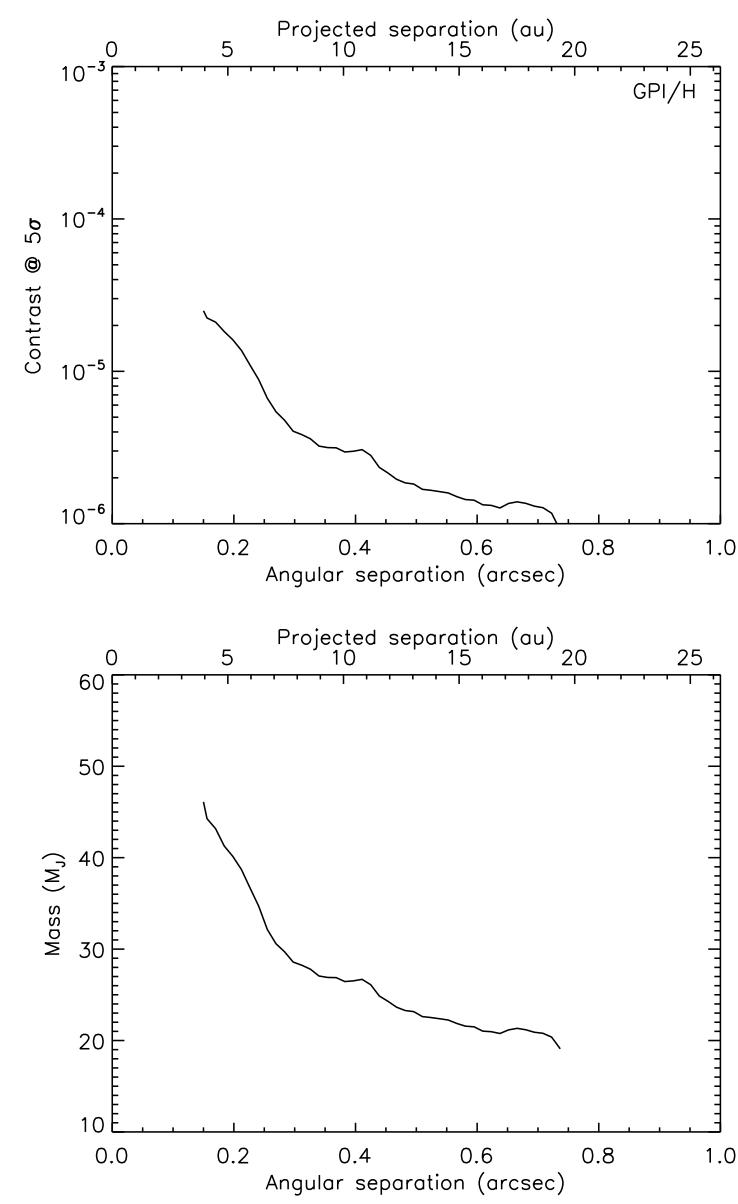

Fig. F.1. GPI $5 \sigma$ detection limits of HD 72945 in contrast with respect to the star (top) and the planet mass (bottom). 